Quim. Nova, Vol. 36, No. 4, 528-532, 2013

\title{
DETERMINAÇÃO DA CONSTANTE DE VELOCIDADE ABSOLUTA PARA A REAÇÃO DE ABSTRAÇÃO DE HIDROGÊNIO FENÓLICO PELO ESTADO EXCITADO TRIPLETE DE XANTONA EM ACETONITRILA E NO LÍQUIDO IÔNICO HEXAFLUORFOSFATO DE 1-BUTIL-3-METIL-IMIDAZÓLIO [bmim.PF ${ }_{6}$ ]
}

\author{
Ada Ruth Bertoti \\ Departamento de Química Geral e Inorgânica, Instituto de Química, Universidade Federal da Bahia, Campus de Ondina, \\ 40170-490 Salvador - BA, Brasil \\ José Carlos Netto-Ferreira* \\ Departamento de Química Geral e Inorgânica, Instituto de Química, Universidade Federal da Bahia, Campus de Ondina, \\ 40170-490 Salvador - BA / Departamento de Química, Instituto de Ciências Exatas, Universidade Federal Rural do Rio de Janeiro, \\ 21970-000 Seropédica - RJ, Brasil
}

Recebido em 17/7/12; aceito em 31/10/12; publicado na web em 18/2/13

\begin{abstract}
DETERMINATION OF ABSOLUTE RATE CONSTANT FOR THE PHENOLIC HYDROGEN ABSTRACTION REACTION BY THE TRIPLET EXCITED STATE OF XANTHONE IN ACETONITRILE AND IN IONIC LIQUID 1-BUTYL-3METHYLIMIDAZOLIUM HEXAFLUOROPHOSPHATE [bmim.PF $]$ ]. The triplet excited state of xanthone was generated and characterized by laser flash photolysis in acetonitrile $\left(\lambda_{\max }=620 \mathrm{~nm} ; \tau=1.8 \mu \mathrm{s}\right)$ and in ionic liquid 1-butyl-3-methylimidazolium hexafluorophosphate $\left[\mathrm{bmim} . \mathrm{PF}_{6}\right]\left(\lambda_{\max }=620 \mathrm{~nm} ; \tau=3.0 \mu \mathrm{s}\right)$. It reacts with phenols yielding the corresponding xanthone ketyl radical. Stern-Volmer plots for the reaction of triplet xanthone with phenols led to the determination of absolute rate constants for phenolic hydrogen abstraction in the order of $\sim 10^{9} \mathrm{Lmol}^{-1} \mathrm{~s}^{-1}$ in acetonitrile and $\sim 10^{8} \mathrm{Lmol}^{-1} \mathrm{~s}^{-1}$ in $\left[\mathrm{bmim} . \mathrm{PF}_{6}\right]$. The lower diffusioncontrolled rate constant for $\left[\mathrm{bmim} . \mathrm{PF}_{6}\right]$ is responsible for the difference in the phenolic hydrogen abstraction rate constants in this solvent.
\end{abstract}

Keywords: xanthone; ionic liquid; triplet excited state.

\section{INTRODUÇÃO}

O estado excitado triplete de cetonas aromáticas tem sido extensivamente estudado, não só do ponto de vista experimental, como também teórico, face à sua importância tanto em fotoquímica como em fotobiologia. ${ }^{1-7}$

As energias dos estados excitados singlete e triplete de carbonilas, como também as suas propriedades fotofísicas e fotoquímicas, são fortemente influenciadas pela polaridade do solvente. Para cetonas aromáticas como xantona, que apresentam uma diferença de energia pequena entre os seus estados $n \pi^{*}$ e $\pi \pi^{*}$, o grau de mistura entre estes dois estados é significativo e, de forma geral, o aumento da polaridade do solvente resulta na estabilização do estado excitado de configuração $\pi \pi^{*}$ e na desestabilização do estado $n \pi^{*}$. Portanto, em solventes polares, xantona apresenta estado excitado de energia mais baixa com caráter $\pi \pi^{*}$, enquanto que em solventes apolares o triplete de energia mais baixa tem caráter $n \pi * .8$

O momento de dipolo para o estado excitado triplete de xantona, em solvente apolar, mede 3,51 D, ${ }^{9}$ enquanto para o estado fundamental é de 3,10 D. ${ }^{10}$ Entretanto, é razoável se esperar que haja uma mudança nos momentos dipolares para os estados fundamental e excitado triplete, mesmo em fase aquosa. ${ }^{11}$ Devido a estas características, ou seja, mudança da configuração do estado excitado como uma função da polaridade do solvente e variação do momento dipolar após o processo de excitação eletrônica, xantona tem sido freqüentemente empregada como sensor de polaridade do ambiente micro-heterogêneo em complexos supramoleculares formados por micelas, ${ }^{12-17}$ ciclodextrinas, ${ }^{18-23}$ membranas de Nafion ${ }^{24}$ e zeólitas. ${ }^{25-27}$

Além disso, uma vez que o estado excitado triplete $\pi \pi^{*}$ de xantona é muito pouco reativo e a posição da absorção triplete-triplete

*e-mail: jcnetto@ufrrj.br varia com a polaridade do solvente, é possível seguir diretamente a realocação do seu triplete, permitindo assim a determinação das suas constantes de velocidade de entrada e saída da cavidade em sistemas supramoleculares. ${ }^{17,18}$

A reatividade do estado excitado triplete de xantona $\left(\lambda_{\max }=630 \mathrm{~nm}\right.$ e $\tau=1,8 \mu \mathrm{s}$, em ACN) ${ }^{28}$ tem sido exaustivamente estudada. Estudos por fotólise por pulso de laser mostraram que a irradiação de xantona em presença de supressores que têm a capacidade de doar elétron, como aminas, resulta na formação do seu ânion radical, ${ }^{29}$ enquanto que em presença de doadores de hidrogênio, tais como alcoóis, hidreto de tributil estanho, indol e alguns fenóis, observa-se a formação do radical cetila correspondente..$^{28-30}$

Por outro lado, a excitação de xantona no líquido iônico [bmim. $\mathrm{PF}_{6}$ ] revelou a formação do seu estado excitado triplete com $\lambda_{\text {max }}$ a 622 nm e tempo de vida de 4,5 $\mu \mathrm{s} .{ }^{31}$ Este triplete reage por transferência de energia com naftaleno $\left(\mathrm{k}_{\mathrm{q}}=1,1 \times 10^{8} \mathrm{~L} \mathrm{~mol}^{-1} \mathrm{~s}^{-1}\right)$ e por transferência de hidrogênio com difenilmetano $\left(\mathrm{k}_{\mathrm{q}}=6,8 \times 10^{6} \mathrm{~L} \mathrm{~mol}^{-1} \mathrm{~s}^{-1}\right) .{ }^{31}$

Ao longo dos últimos anos o nosso grupo de pesquisa tem dedicado um esforço considerável no estudo do mecanismo de abstração de hidrogênio fenólico por parte do estado excitado triplete de compostos aromáticos mono- e dicarbonilados. ${ }^{32-39}$ Estudos por fotólise por pulso de laser de nanossegundo, assim como cálculos teóricos de DFT para a reação de cetonas e $\alpha$-dicetonas com fenóis nos levaram a concluir que, diferentemente do mecanismo conhecido como tipo alcoxila operante na abstração de hidrogênio alquílico, para o caso de fenóis como doadores de hidrogênio a reação se passa via uma transferência acoplada de elétron e próton (PCeT). ${ }^{40}$ Para xantona podem ser encontrados na literatura alguns poucos valores para a constante de abstração de hidrogênio fenólico, ${ }^{29,30}$ não havendo, entretanto, nenhum estudo sistemático do efeito de fenóis contendo substituintes polares sobre esta constante, tanto em solventes orgânicos convencionais quanto em líquidos iônicos. 
Neste trabalho são apresentados dados cinéticos, empregando-se a técnica de fotólise por pulso de laser de nanossegundo, acerca da reação do triplete de xantona (1) com fenóis contendo substituintes polares, em acetonitrila. Estes dados são comparados àqueles obtidos quando se empregou como solvente o líquido iônico hexafluorfosfato de 1-butil-3-metilimidazólio, [bmim.PF ${ }_{6}$.
(1)<smiles>O=c1c2ccccc2oc2ccccc12</smiles>

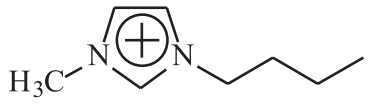

[bmim.. $\left.\mathrm{PF}_{6}\right]$

\section{PARTE EXPERIMENTAL}

\section{Reagentes}

Acetonitrila grau HPLC foi adquirida de J. T. Baker. Hexafluorfosfato de 1-n-butil-3-metilimidazólio é um produto sintetizado e purificado nos laboratórios da Universidade Federal do Rio Grande do Sul e foi adquirido junto à Fundação Universidade Federal do Rio Grande do Sul (FURGS). A principal característica que nos levou à sua aquisição foi o fato de que, diferentemente dos outros líquidos iônicos encontrados no mercado, apresenta excelente pureza espectroscópica a $355 \mathrm{~nm}(\mathrm{~A}=0,0)$, comprimento de onda de excitação empregado neste trabalho. A xantona foi adquirida da Aldrich e recristalizada em etanol antes de usada. Os fenóis foram adquiridos da Aldrich, apresentando grau de pureza superior a $95 \%$, e usados como recebidos.

\section{Equipamentos}

Os espectros na região do ultravioleta foram obtidos em um espectrofotômetro Hewlett Packard 8453 com detector de conjunto de iodos, interfaciado com um computador pessoal contendo o programa Windows Workstation 4.0.

Os experimentos de fotólise por pulso de laser em nanossegundos foram realizados utilizando um sistema Luzchem modelo MLFP 112 empregando uma lâmpada de xenônio Cermax de $175 \mathrm{~W}$, um monocromador da CUI laser Corporation Digikröm CM 110, uma fotomultiplicadora Hamamatsu e um osciloscópio Tektronix modelo TDS 2012, onde os sinais da fotomultiplicadora são digitalizados e transmitidos para um computador (Dell, Pentium IV $3.4 \mathrm{GHz}$ ) por meio de uma interface GPIB.

As amostras foram irradiadas com um laser Nd/YAG da Continuum, modelo Surelite II, utilizando o terceiro harmônico ( $\lambda=355 \mathrm{~nm}$, pulso de $10 \mathrm{~ns}, \sim 40 \mathrm{~mJ} /$ pulso). O feixe, pulsado continuamente a uma frequência de $1 \mathrm{~Hz}$, é concentrado, porém não focalizado no compartimento da amostra. Em todos os experimentos foi utilizada uma cela de quartzo estática 10 x $10 \mathrm{~mm}$, contendo 1 $\mathrm{mL}$ (para o líquido iônico) ou $3 \mathrm{~mL}$ (para acetonitrila) de solução de xantona, que foi desaerada por $30 \mathrm{~min}$ com argônio. A concentração da cetona $\left(\sim 10^{-3} \mathrm{~mol} \mathrm{~L}^{-1}\right)$ foi ajustada de forma a fornecer uma absorbância de $\sim 0,3$ no comprimento de onda de excitação $(355 \mathrm{~nm}$ ).

As constantes de velocidade para a reação do triplete de xantona frente aos diferentes supressores foram obtidas a partir de gráficos de Stern-Volmer, ${ }^{41}$ segundo a Equação 1 , tendo-se obtido gráficos lineares em todos os casos:

$$
\mathrm{k}_{\mathrm{obs}}=\mathrm{k}_{\mathrm{o}}+\mathrm{k}_{\mathrm{q}}[\mathrm{Q}]
$$

onde: $\mathrm{k}_{\mathrm{o}}$ é a constante de velocidade de decaimento para o triplete de xantona na ausência de supressor; $\mathrm{k}_{\mathrm{q}}$ é a constante de velocidade de decaimento para o triplete de xantona na presença de supressor e [Q] é a concentração do supressor expressa em $\mathrm{mol} \mathrm{L}^{-1}$.

Nos experimentos de supressão foram preparadas soluções estoque dos supressores de modo que, para obter as concentrações apropriadas do supressor, foi necessário somente adicionar microlitro(s) da solução estoque à célula contendo a solução da cetona.

\section{RESULTADOS E DISCUSSÃO}

A fotólise por pulso de laser $\left(\lambda_{\text {exc }}=355 \mathrm{~nm}\right)$ de uma solução de xantona em acetonitrila resultou na formação de um transiente com máximo de absorção a $620 \mathrm{~nm}$, apresentando tempo de vida de $1,8 \mu \mathrm{s}$, o qual foi associado ao seu estado excitado triplete, de acordo com dados existentes na literatura ${ }^{28}$ (Figura 1). Empregando trifluoretanol como solvente, a absorção máxima sofre um deslocamento considerável para o azul, com $\lambda_{\text {max }}$ a $580 \mathrm{~nm}$ (Figura 1), o que está completamente em acordo com a literatura, uma vez que é sabido que o triplete de xantona é extremamente dependente da polaridade do solvente. ${ }^{28}$

A característica triplete deste transiente foi confirmada pela supressão com 1-metilnaftaleno $\left(\mathrm{E}_{\mathrm{T}}=60,7 \mathrm{kcal} \mathrm{mol}^{-1}\right),{ }^{42}$ conhecido supressor de triplete por transferência de energia, para o qual foi encontrada, em acetonitrila, uma constante de velocidade de supressão controlada por difusão, isto é, $(1,6 \pm 0,1) \times 10^{10} \mathrm{~L} \mathrm{~mol}^{-1} \mathrm{~s}^{-1}$ (Figura $1 \mathrm{~S}$, material suplementar). Além disso, o espectro do transiente observado após todo o triplete de xantona ter sido suprimido é consistente com o observado na literatura para o triplete de 1 -metilnaftaleno $\left(\lambda_{\max }=\right.$ $410 \mathrm{~nm}){ }^{42}$ (Figura 2).

O estado excitado triplete de xantona é suprimido eficientemente por doadores de hidrogênio, como fenol e seus derivados contendo substituintes polares no anel aromático, como mostram os dados apresentados na Figura 3 e Tabela 1. Os gráficos de Stern-Volmer para o estudo de supressão em acetonitrila foram todos lineares e estão apresentados nas Figuras 2S-17S, material suplementar.

Uma inspeção da Tabela 1 mostra claramente que as constantes de velocidade de supressão do triplete de xantona por fenol e seus derivados contendo substituintes polares, em acetonitrila, são quase que independentes do substituinte e muito próximas ao valor da constante de velocidade de difusão neste solvente, sendo em alguns casos idêntica (como para 4-metoxifenol e hidroquinona). Isto demonstra uma quase que completa ausência de seletividade no processo de abstração de hidrogênio quando fenóis são empregados como supressores.

A alta eficiência de supressão do triplete de cetonas aromáticas por fenóis é consequência da baixa energia de dissociação da ligação O-H de fenóis, bem como devido ao seu baixo potencial de oxidação. ${ }^{43}$ Assim, o mecanismo proposto no processo de abstração de hidrogênio fenólico por xantona envolve a formação de um exciplexo triplete, estabilizado por ponte de hidrogênio, seguida por uma transferência acoplada elétron/próton, levando ao par de radicais cetila/fenoxila como produtos finais (Esquema 1)..$^{30,32-39,44-48}$ Tal mecanismo tem sido recentemente corroborado por cálculos do tipo DFT através dos quais foi claramente demonstrado que a eletrofilicidade da cetona no estado excitado triplete é a força motriz para o processo de abstração de hidrogênio fenólico. 4,6,7,38,39

A irradiação a $355 \mathrm{~nm}$ de uma solução de xantona em acetonitrila quando em presença de excesso de fenol, ou de seus derivados contendo substituintes polares, levou à formação de um novo transiente com absorções máximas a 380, 480 e 580 nm (Figura 4), atribuídas ao radical cetila derivado da xantona. ${ }^{28} \mathrm{~A}$ absorção a 380 $\mathrm{nm}$ pode conter uma contribuição do radical fenoxila, que apresenta absorção não muito intensa na região de 385-405 nm, dependendo 

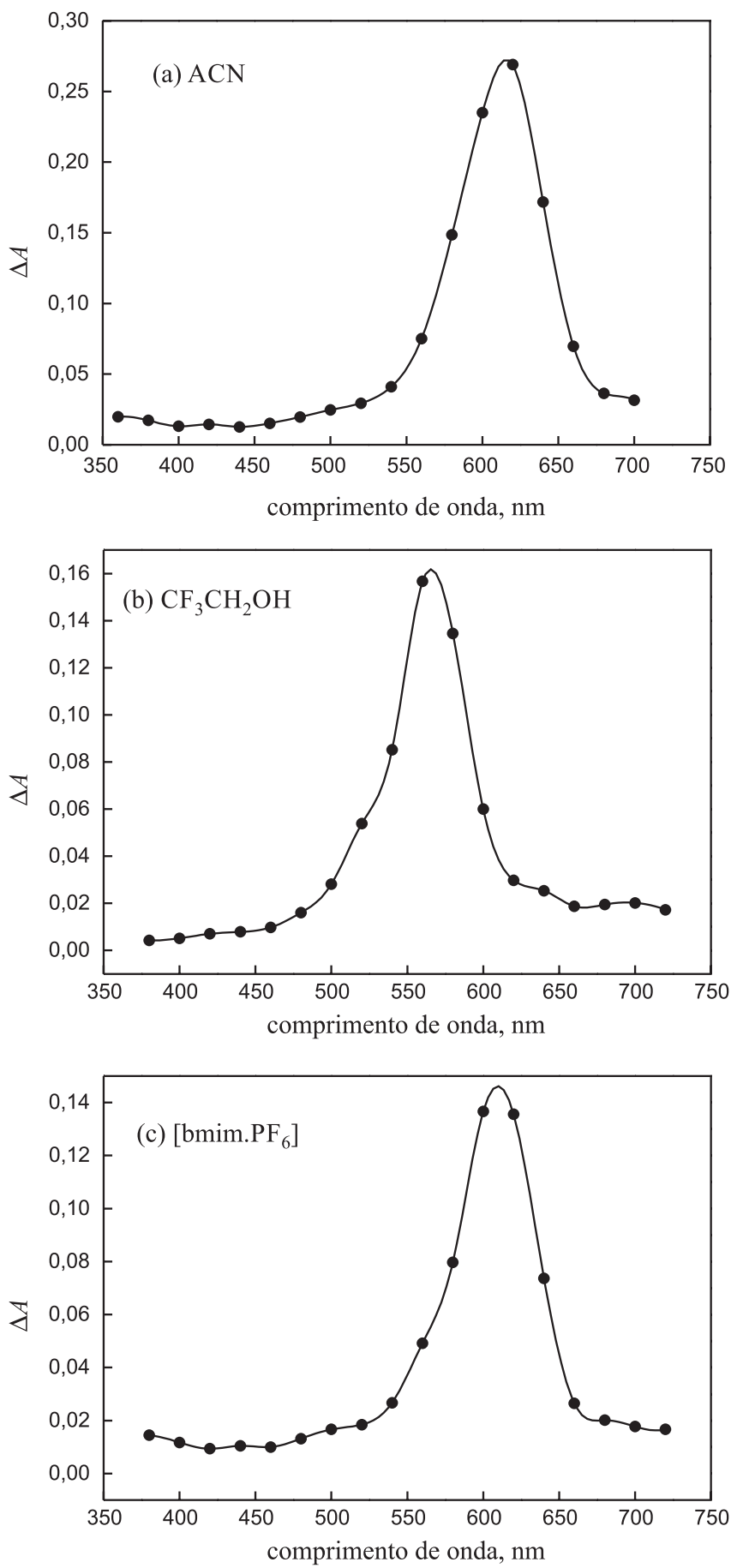

Figura 1. Espectros de absorção para o transiente gerado na excitação de xantona $\left(\lambda_{\text {exc }}=355 \mathrm{~nm}\right)$, em (a) acetonitrila $(A C N)$, (b) 2,2,2-trifluoretanol $e(c)$ [bmim. $P F_{6}$ ], registrados, respectivamente, a 1,1; 3,2 e 1,0 $\mu$ s após o pulso do laser

do substituinte. ${ }^{49-51}$ Uma comparação entre os espectros mostrados nas Figuras 1 (estado excitado triplete da xantona) e 4A (radical cetila derivado da xantona) indica que as absorções observadas para este último são bem menos intensas do que aquela correspondente ao do seu estado excitado triplete, consistente com o reportado na literatura. ${ }^{28,29}$

A excitação com o $3^{\circ}$ harmônico de um laser de Nd/YAG (355 $\mathrm{nm}$ ) de uma solução de xantona em [bmim. $\left.\mathrm{PF}_{6}\right]$ levou à formação de um transiente com absorção máxima a $620 \mathrm{~nm}$, o qual decai com uma cinética de primeira ordem e tempo de vida de $3 \mu$ s (Figura 1). O

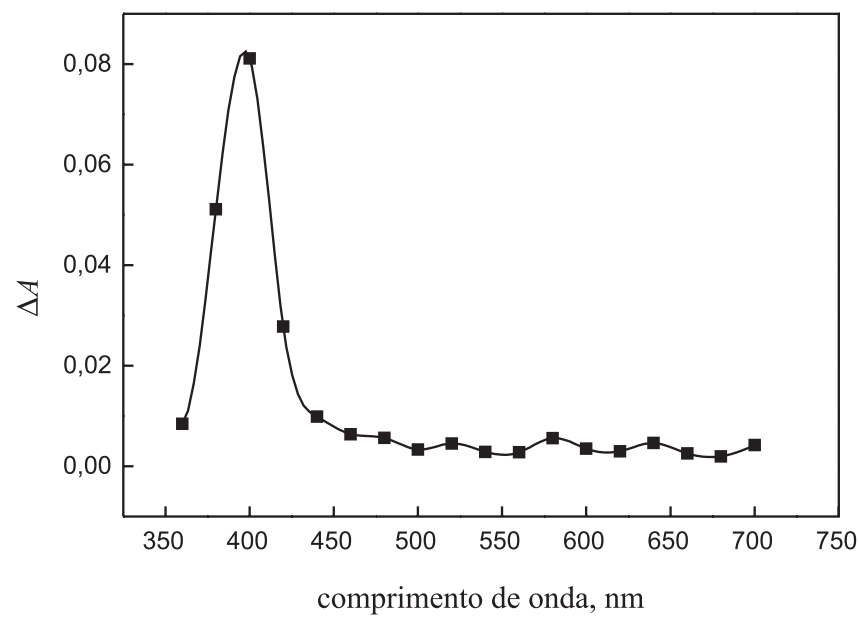

Figura 2. Espectro de absorção para o transiente gerado na excitação de xantona em presença de $2,3 \times 10^{-3} \mathrm{~mol} \mathrm{~L}^{-1}$ de 1-metilnaftaleno, em acetonitrila, registrado $1,7 \mu$ s após o pulso do laser

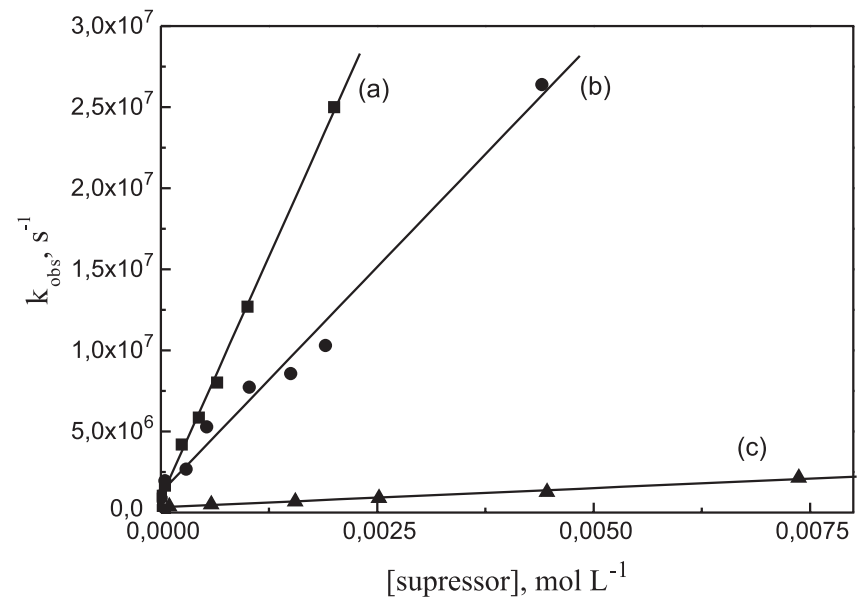

Figura 3. Gráficos representativos de Stern-Volmer para a supressão do triplete de xantona por fenóis: (a) hidroquinona em acetonitrila; (b) 4-metilfenol em acetonitrila e (c) fenol em [bmim.P $F_{6}$ ]

Tabela 1. Constantes de velocidade de segunda ordem para a reação do estado excitado triplete de xantona com fenóis, em acetonitrila e em [bmim. $\left.\mathrm{PF}_{6}\right]$

\begin{tabular}{lcc}
\hline Supressor & $\begin{array}{c}\mathrm{k}_{\mathrm{q}}, \mathrm{L} \mathrm{mol}^{-1} \mathrm{~s}^{-1} \\
(\mathrm{ACN})\end{array}$ & $\begin{array}{c}\mathrm{k}_{\mathrm{q}}, \mathrm{L} \mathrm{mol}^{-1} \mathrm{~s}^{-1} \\
\left(\left[\mathrm{bmim} \cdot \mathrm{PF}_{6}\right]\right)\end{array}$ \\
\hline $\begin{array}{l}(3,1 \pm 0,1) \times 10^{9} \\
\text { fenol }\end{array}$ & $(2,3 \pm 0,1) \times 10^{8}$ \\
4-fluorfenol & $(4,4 \pm 0,2) \times 10^{9}$ & -- \\
4-metoxifenol & $(1,3 \pm 0,3) \times 10^{10}$ & $(3,6 \pm 0,1) \times 10^{8}$ \\
hidroquinona & $(1,1 \pm 0,4) \times 10^{10}$ & -- \\
4-cianofenol & $(3,8 \pm 0,2) \times 10^{9}$ & -- \\
4-terc-butilfenol & $(3,7 \pm 0,9) \times 10^{9}$ & $(1,7 \pm 0,1) \times 10^{8}$ \\
4-bromofenol & $(4,6 \pm 0,1) \times 10^{9}$ & -- \\
4-clorofenol & $(4,3 \pm 0,3) \times 10^{9}$ & $(2,2 \pm 0,1) \times 10^{8}$ \\
4-metilfenol & $(5,6 \pm 0,3) \times 10^{9}$ & $(2,3 \pm 0,2) \times 10^{8}$ \\
4-fenilfenol & $(8,0 \pm 0,3) \times 10^{9}$ & $(2,1 \pm 0,2) \times 10^{8}$ \\
3-clorofenol & $(6,8 \pm 0,2) \times 10^{9}$ & -- \\
3-metilfenol & $(6,3 \pm 0,2) \times 10^{9}$ & -- \\
3-metoxifenol & $(3,7 \pm 0,1) \times 10^{9}$ & -- \\
3-fluorfenol & $(2,9 \pm 0,1) \times 10^{9}$ & - \\
resorcinol & $(4,5 \pm 0,3) \times 10^{9}$ & -- \\
3-bromofenol & $(5,5 \pm 0,3) \times 10^{9}$ & -- \\
\hline
\end{tabular}




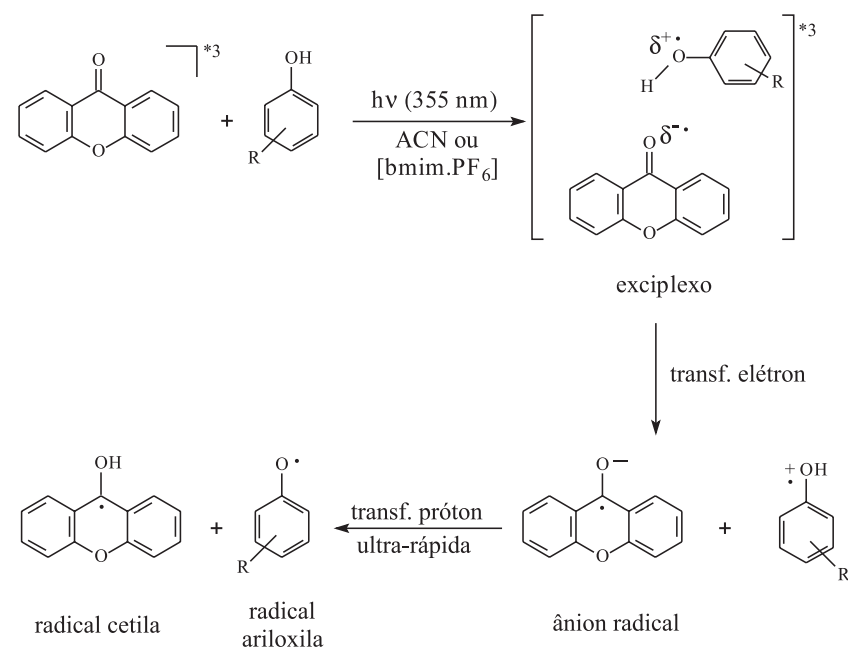

Esquema 1. Mecanismo proposto para a abstração de hidrogênio fenólico por xantona
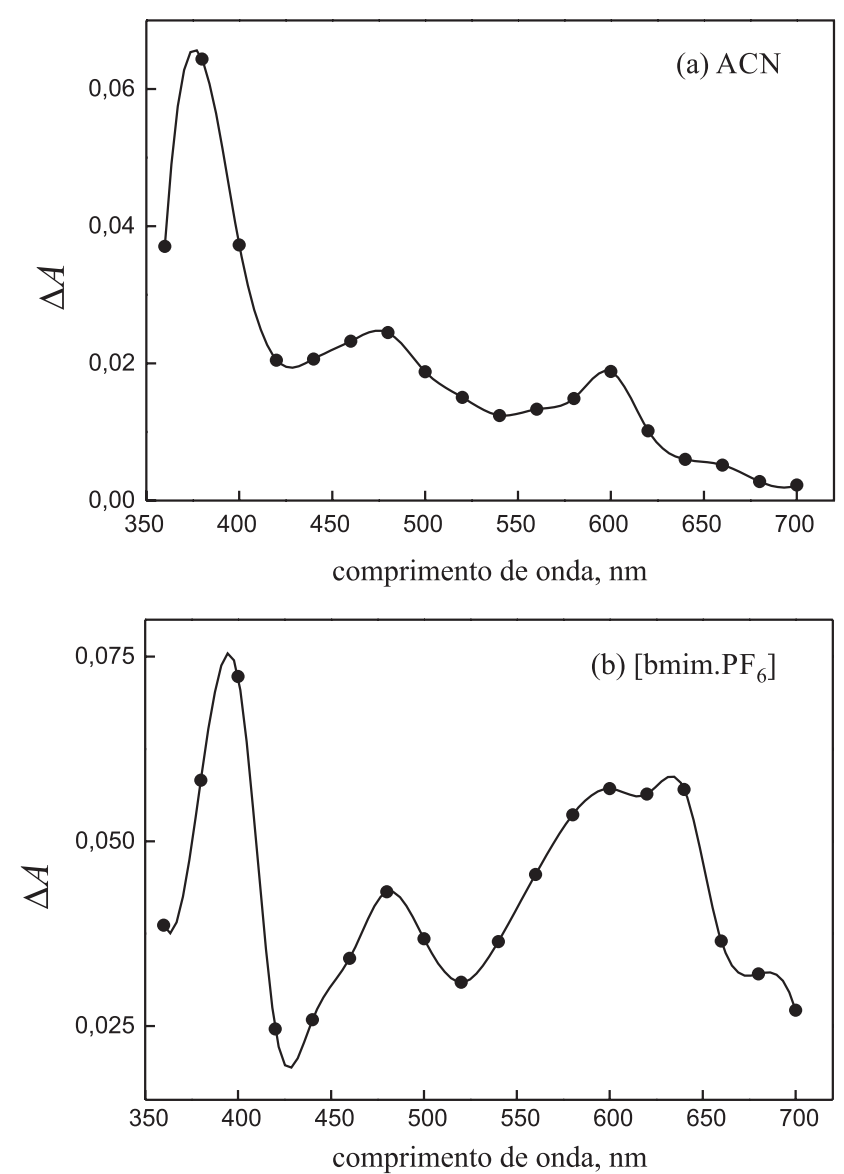

Figura 4. Espectro de absorção para os transientes gerados na fotólise de xantona: (a) com 5,2 $\times 10^{-3} \mathrm{~mol} \mathrm{~L}^{-1}$ de hidroquinona, em acetonitrila e (b) com 5,6 $\times 10^{-3}$ mol L $^{-1}$ de 4-metoxifenol, em [bmim.PF $F_{6}$. Ambos os espectros foram registrados $1,7 \mu$ s após o pulso do laser

valor para o máximo de absorção para este solvente é absolutamente coincidente com o encontrado para acetonitrila, o que demonstra claramente que a polaridade do líquido iônico $\left[\mathrm{bmim}_{.} \mathrm{PF}_{6}\right]$ deve ser idêntica à daquele solvente $\left(\lambda_{\max }{ }^{\mathrm{T}-\mathrm{T}}=620 \mathrm{~nm}\right)$ e menor do que para 2,2,2-trifluoretanol $\left(\lambda_{\max }^{\mathrm{T}-\mathrm{T}}=580 \mathrm{~nm}\right)$. O maior tempo de vida para o triplete da xantona em [bmim. $\left.\mathrm{PF}_{6}\right]$ está associado à maior viscosidade do solvente, uma vez que, neste caso, modos de desativação do triplete por encontros bimoleculares, como a aniquilação triplete-triplete, são menos prováveis. ${ }^{52}$ Novamente, a confirmação do caráter triplete deste transiente foi feita utilizando-se 1-metilnaftaleno como supressor de triplete por transferência de energia, tendo-se obtido uma constante de velocidade de supressão de $(3,2 \pm 0,2) \times 10^{8} \mathrm{~L} \mathrm{~mol}^{-1} \mathrm{~s}^{-1}$, idêntica à constante de velocidade de difusão em $\left[b_{\text {mim. }} . \mathrm{PF}_{6}\right] .{ }^{52}$ É importante assinalar que o valor da constante de velocidade no limite difusional, o qual pode ser determinado segundo a Equação de Smoluchowski, ${ }^{42}$ é inversamente proporcional à viscosidade do solvente. Devido à alta viscosidade dos líquidos iônicos, esta constante de velocidade é de cerca de duas ordens de grandeza menor do que as observadas para solventes orgânicos convencionais. ${ }^{53}$

$\mathrm{Na}$ Tabela 1 encontram-se listados os valores de $\mathrm{k}_{\mathrm{q}}$ obtidos para a supressão de xantona em [bmim.PF6] por fenol, 4-metoxifenol, 4-terc-butilfenol, 4-fenilfenol, 4-metilfenol e 4-clorofenol. A supressão do estado excitado triplete de xantona por fenol e seus derivados contendo substituintes polares, em [bmim. $\left.\mathrm{PF}_{6}\right]$, apresenta uma constante de velocidade controlada pela difusão em todos os casos, isto é, de cerca de $10^{8} \mathrm{~L} \mathrm{~mol}^{-1} \mathrm{~s}^{-1}$, com os gráficos de Stern-Volmer para esta supressão sendo lineares em todos os casos, os quais são apresentados nas Figuras 18S-23S, material suplementar.

Estes valores, independentes do substituinte, mostram que o mecanismo de supressão por transferência de hidrogênio fenólico para o triplete de xantona neste solvente não apresenta nenhuma seletividade, sendo controlado única e exclusivamente pela probabilidade de colisão entre estado excitado e supressor. Este comportamento é semelhante ao encontrado para xantona em acetonitrila, como descrito acima, uma vez que, neste solvente, as constantes de velocidade de supressão também foram independentes do substituinte.

De maneira similar ao observado quando se utilizou acetonitrila como solvente, a irradiação a $355 \mathrm{~nm}$ de uma solução de xantona em [bmim. $\mathrm{PF}_{6}$ ], quando em presença de excesso dos fenóis empregados nos estudos de supressão, também levou à formação de um novo transiente com absorções máximas a 380, 480 e 600 nm (Figura 4B), as quais podem ser associadas ao radical cetila derivado da xantona. ${ }^{28}$ Neste caso, observa-se também a presença de uma absorção de baixa intensidade a $620 \mathrm{~nm}$, que pode ser atribuída ao estado excitado triplete da xantona não reagida.

\section{CONCLUSÕES}

A irradiação de xantona em acetonitrila $\left(\lambda_{\max }=620 \mathrm{~nm}\right.$ e $\tau_{\mathrm{T}}=1,8$ $\mu$ ) levou à formação do seu estado excitado triplete, caracterizado por estudos de supressão com 1-metilnaftaleno. Comportamento similar foi observado quando se empregou como solvente o líquido iônico [bmim. $\mathrm{PF}_{6}$ ], tendo-se encontrado, neste caso, $\lambda_{\max }=620 \mathrm{~nm} \mathrm{e}$ $\tau_{\mathrm{T}}=3,0 \mu \mathrm{s}$. As constantes de velocidade de abstração de hidrogênio frente a fenóis contendo substituintes polares são independentes do substituinte em ambos os solventes e, em todos os casos, foi observada a formação do radical cetila derivado da xantona após a reação com os fenóis.

\section{MATERIAL SUPLEMENTAR}

Disponibilizado em http://quimicanova.sbq.org.br, em arquivo pdf, com acesso livre.

\section{AGRADECIMENTOS}

Este trabalho foi financiado pela FAPESB, CNPq e FINEP. A. R. Bertoti agradece ao CNPq a bolsa de doutoramento concedida. J. C. Netto-Ferreira agradece ao $\mathrm{CNPq}$ a bolsa de Pesquisador Visitante na Universidade Federal da Bahia. 


\section{REFERÊNCIAS}

1. Yoshihara, T.; Yamaji, M.; Itoh, T.; Shizuka, H.; Shimokage, T.; TeroKubota, S.; Phys. Chem. Chem. Phys. 2000, $2,993$.

2. Scaiano, J. C.; J. Photochem. 1973/74, 2, 81.

3. Adam, W.; Andler, S.; Saha-Möller, C. R.; Arch. Biochem. Biophys. 1998, 349, 261.

4. Pan, Y.; Fu, Y.; Liu, S.; Yu, H.; Gao, Y.; Guo, Q.; Yu, S.; J. Phys. Chem. A 2006, 110, 7316.

5. Takaizumi, A. A. C.; Santos, F. R.; Silva, M. T.; Netto-Ferreira, J. C.; Quim. Nova 2009, 32, 1799.

6. Perez-Prieto, J.; Stiriba, S.-E.; Boscá, F.; Lahoz, A.; Domingo, L. R.; Mourabit, F.; Monti, S.; Miranda, M. A.; J. Org. Chem. 2004, 69, 8618.

7. Perez-Prieto, J.; Boscá, F.; Galian, R. E.; Lahoz, A.; Domingo, L. R.; Miranda, M. A.; J. Org. Chem. 2003, 68, 5104.

8. Ley, C.; Morlet-Savary, F.; Fouassier, J. P.; Jacques, P.; J. Photochem. Photobiol., A 2000, 137, 87.

9. Abdullah, K. A.; Kemp, T. J.; J. Photochem. 1986, 32, 49.

10. Mc Clellan, A. L.; Tables of Experimental Dipole Moments, Freeman: San Francisco, 1963.

11. Abuin, E. B.; Scaiano, J. C.; J. Am. Chem. Soc. 1984, 106, 6274.

12. Scaiano, J. C.; Selwyn, L. C.; Can. J. Chem. 1981, 59, 2368.

13. Barra, M.; Supramol. Chem. 1997, 8, 263.

14. Murphy, R. S.; Barros, T. C.; Barnes, J.; Mayer, B.; Marconi, G.; Bohne, C.; J. Phys. Chem. A 1999, 103, 137.

15. Barra, M.; Bohne, C.; Scaiano, J. C.; Photochem. Photobiol. 1991, 54, 1.

16. Barra, M.; Scaiano, J. C.; Photochem. Photobiol. 1995, 62, 60.

17. Mohtat, N.; Cozens, F. L.; Scaiano, J. C.; J. Phys. Chem. B 1998, 102, 7557.

18. Bohne, C.; Barra, M.; Boch, R.; Abuin, E. B.; Scaiano, J. C.; J. Photochem. Photobiol., A 1992, 65, 249.

19. Barra, M.; Bohne, C.; Scaiano, J. C.; J. Am. Chem. Soc. 1990, 112, 8075.

20. Liao, Y.; Frank, J.; Holzwarth, J. F.; Bohne, C.; Chem. Commun. 1995, 199.

21. Liao, Y.; Bohne, C.; J. Phys. Chem. 1996, 100, 734.

22. Song, K.-S.; Hou, C.-R.; Liu, L.; Li, X.-S.; Guoa, Q.-X.; J. Photochem. Photobiol., A 2001, 139, 105.

23. Okano, L. T.; Barros, T. C.; Chou, D. T. H.; Bennet, A. J.; Bohne, C.; J. Phys. Chem. B 2001, 105, 2122.

24. Weir, D.; Scaiano, J. C.; Tetrahedron 1987, 43, 1617.

25. Scaiano, J. C.; de Lucas, N. C.; Andraos, J.; García, H.; Chem. Phys. Lett. 1995, 233, 5.

26. Wilkinson, F.; Willsher, C. J.; Casal, H. L.; Johnston, L. J.; Scaiano, J. C.; Can. J. Chem. 1986, 64, 539.

27. Scaiano, J. C.; Kaila, M.; Corrent, S.; J. Phys. Chem. B 1997, 101, 8564.
28. Scaiano, J. C.; J. Am. Chem. Soc. 1980, 102, 7747.

29. Whang, J.-T.; Pan, Y.; Zhang, L.-M.; Yu, S.-Q.; Chin. J. Chem. Phys. 2007, 20, 396.

30. Lathioor, E. C.; Leigh, W. J.; Photochem. Photobiol. 2006, 82, 291.

31. Alvaro, M.; Ferrer, B.; García, H.; Narayana, M.; Chem. Phys. Lett 2002, $362,435$.

32. Silva, M. T.; Netto-Ferreira, J. C.; J. Photochem. Photobiol., A 2004, 162, 225.

33. Serra, A. C.; de Lucas, N. C.; Netto-Ferreira, J. C.; J. Braz. Chem. Soc. 2004, $15,481$.

34. Netto-Ferreira, J. C.; Silva, E. S. L.; de Lucas, N. C.; J. Photochem. Photobiol., A 2011, 225, 135.

35. de Lucas, N. C.; Fraga, H. S.; Cardoso, C. P.; Corrêa, R. J.; Garden, S. J.; Netto-Ferreira, J. C.; Phys. Chem. Chem. Phys. 2010, 12, 10746.

36. Rodrigues, J. F.; Silva, F. A.; Netto-Ferreira, J. C.; J. Braz. Chem. Soc. 2010, $21,960$.

37. Ribeiro, A. M.; Bertoti, A. R.; Netto-Ferreira, J. C.; J. Braz. Chem. Soc., 2010, 21, 1071 .

38. de Lucas, N. C.; Elias, M.; Firme, C.; Correa, R. J.; Garden, S.; Nicodem, D.; Netto-Ferreira, J. C.; J. Photochem. Photobiol., A 2009, 201, 1.

39. de Lucas, N. C.; Correa, R. J.; Albuquerque, A. C. C.; Firme, C.; Bertoti, A. R.; Netto-Ferreira, J. C.; J. Phys. Chem. A 2007, 111, 1117.

40. Mayer, J. M.; Acc. Chem. Res. 2011, 44, 36.

41. Stern, O.; Volmer, M.; Physik Z 1919, 20, 183.

42. Murov, S. L.; Carmichael, I.; Hug, G. L.; Handbook of Photochemistry, $2^{\text {nd }}$ ed., Marcel Dekker, Inc.: New York, 1993.

43. Das, P. K.; Encinas, M. V.; Scaiano, J. C.; J. Am. Chem. Soc. 1981, 103 , 4154.

44. Biczok, L.; Bérces, T.; Linschitz, H.; J. Am. Chem. Soc. 1997, 119, 11071.

45. de Lucas, N. C.; Silva, M. T.; Gege, C.; Netto-Ferreira, J. C.; J. Chem. Soc., Perkin Trans. 2 1999, 2795.

46. Leigh, W. J.; Lathioor, E. C.; St. Pierre, M. J.; J. Am. Chem. Soc. 1996, $118,12339$.

47. Miranda, M. A.; Lahoz, A.; Martinez-Manez, R.; Boscá, F.; Castell, J. V.; Perez-Prieto, J.; J. Am. Chem. Soc. 1999, 121, 11569.

48. Miranda, M. A.; Lahoz, A.; Boscá, F.; Metni, M. R.; Abdelouahab, F. B.; Castell, J. V.; Pérez-Prieto, J.; Chem. Commun. 2000, 2257.

49. Merényi, G.; Lind, J.; Shen, X.; J. Phys. Chem. 1988, 92, 134.

50. Bansal, K. M.; Fessenden, R. W.; Radiat. Res. 1976, 67, 1.

51. Schuler, R. H.; Neta, P.; Zemel, H.; Fessenden, R. W.; J. Am. Chem. Soc. 1976, $98,3825$.

52. Bertoti, A. R.; Netto-Ferreira, J. C.; Quim. Nova 2009, 32, 1934.

53. McLean, A. J.; Muldoon, M. J.; Gordon, C. M.; Dunkin, I. R.; Chem. Commun. 2002, 1880. 


\section{DETERMINAÇÃO DA CONSTANTE DE VELOCIDADE ABSOLUTA PARA A REAÇÃO DE ABSTRAÇÃO DE HIDROGÊNIO FENÓLICO PELO ESTADO EXCITADO TRIPLETE DE XANTONA EM ACETONITRILA E NO LÍQUIDO IÔNICO HEXAFLUORFOSFATO DE 1-BUTIL-3-METIL-IMIDAZÓLIO [bmim.PF $]$}

\section{Ada Ruth Bertoti}

Departamento de Química Geral e Inorgânica, Instituto de Química, Universidade Federal da Bahia, Campus de Ondina, 40170-490 Salvador - BA, Brasil

José Carlos Netto-Ferreira*

Departamento de Química Geral e Inorgânica, Instituto de Química, Universidade Federal da Bahia, Campus de Ondina, 40170-490 Salvador - BA / Departamento de Química, Instituto de Ciências Exatas, Universidade Federal Rural do Rio de Janeiro, 21970-000 Seropédica - RJ, Brasil

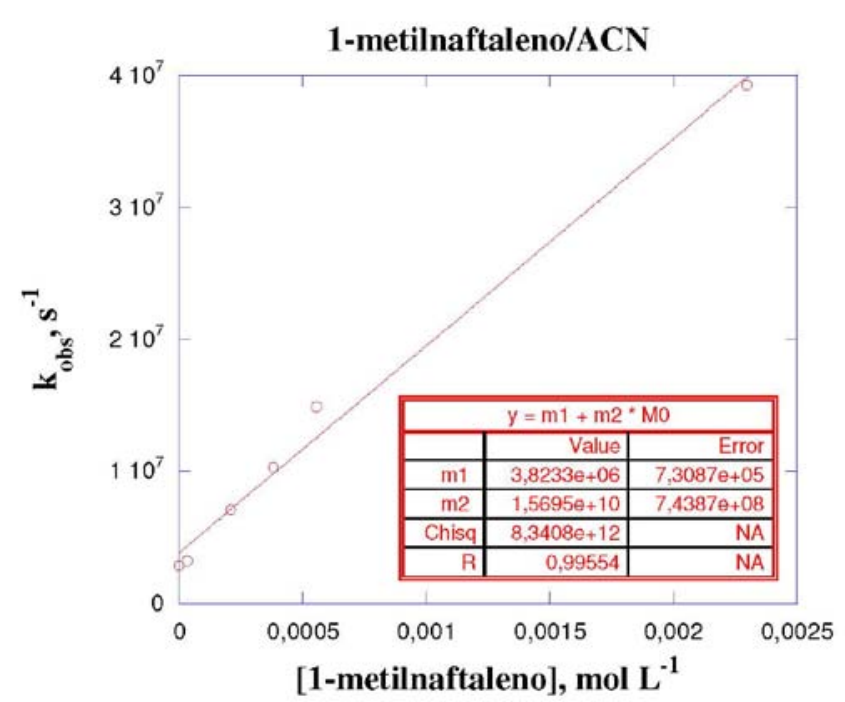

Figura 1S. Gráfico de Stern-Volmer para a supressão do estado excitado triplete de xantona por 1-metilnaftaleno, em acetonitrila

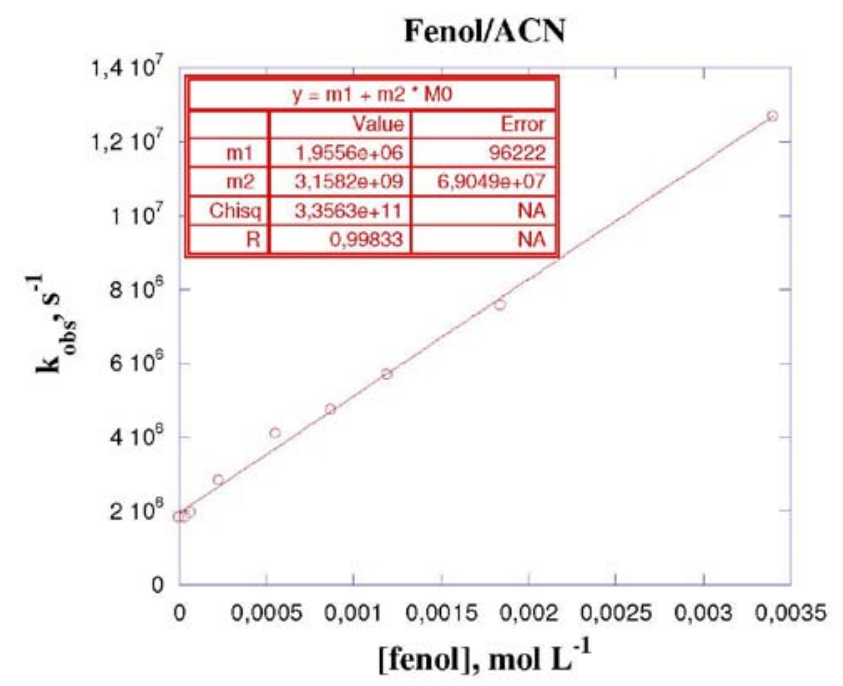

Figura 2S. Gráfico de Stern-Volmer para a supressão do estado excitado triplete de xantona por fenol, em acetonitrila

*e-mail: jcnetto@ufrrj.br

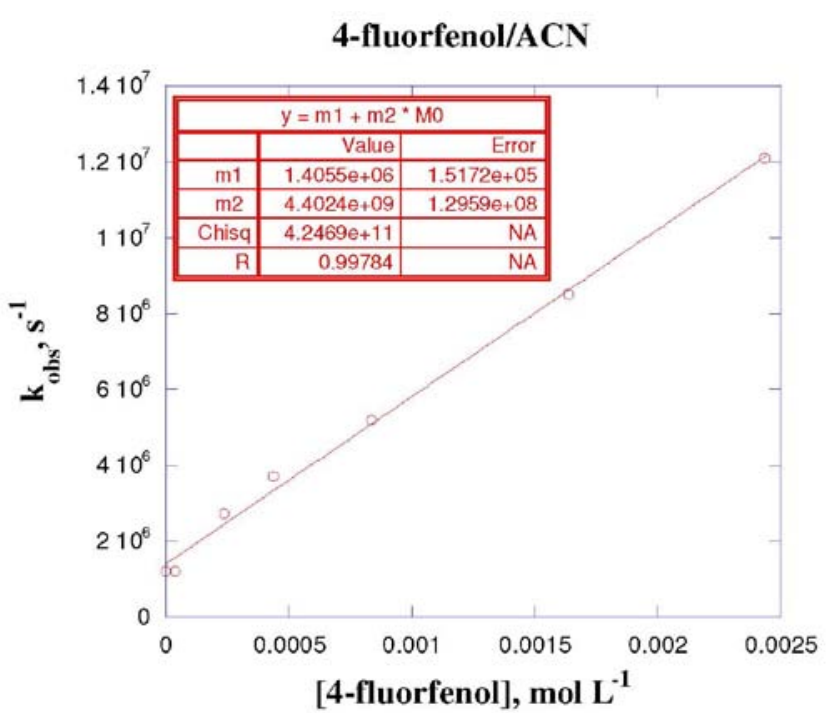

Figura 3S. Gráfico de Stern-Volmer para a supressão do estado excitado triplete de xantona por 4-fluorfenol, em acetonitrila

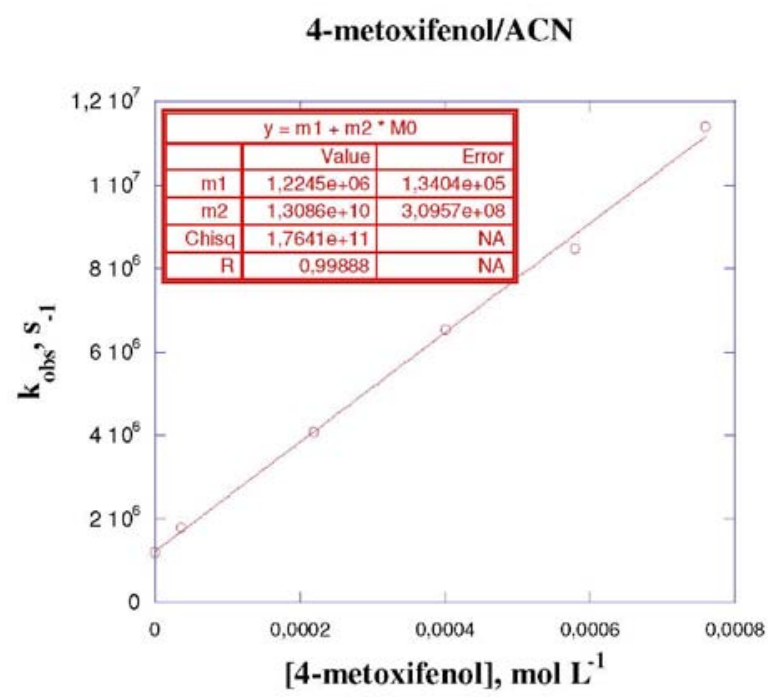

Figura 4S. Gráfico de Stern-Volmer para a supressão do estado excitado triplete de xantona por 4-metoxifenol, em acetonitrila 
hidroquinona/ACN

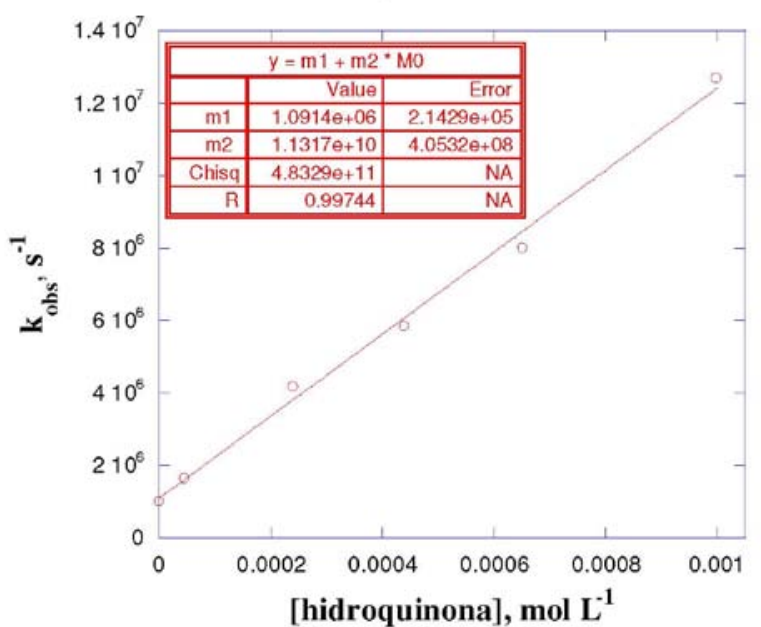

Figura 5S. Gráfico de Stern-Volmer para a supressão do estado excitado triplete de xantona por hidroquinona, em acetonitrila

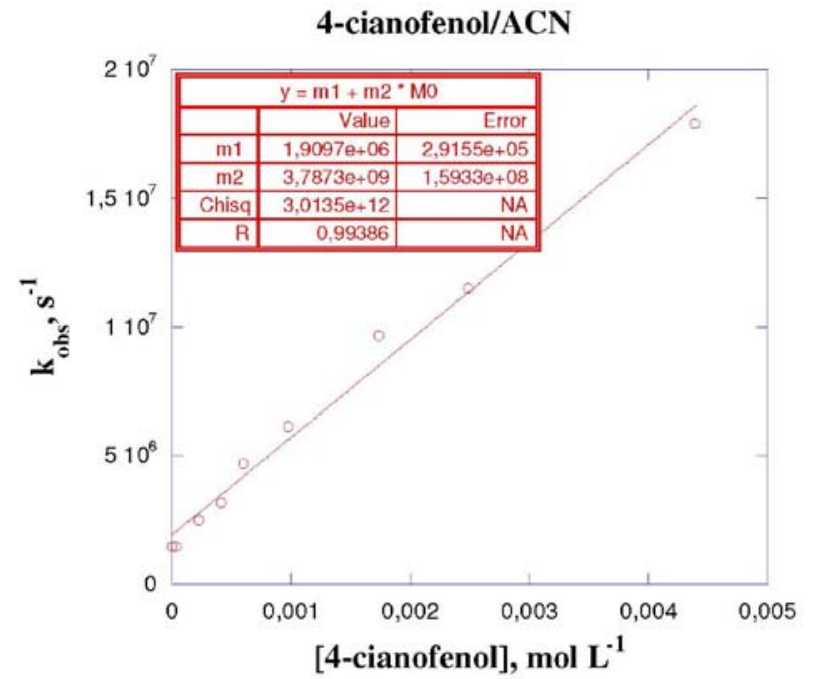

Figura 6S. Gráfico de Stern-Volmer para a supressão do estado excitado triplete de xantona por 4-cianofenol, em acetonitrila

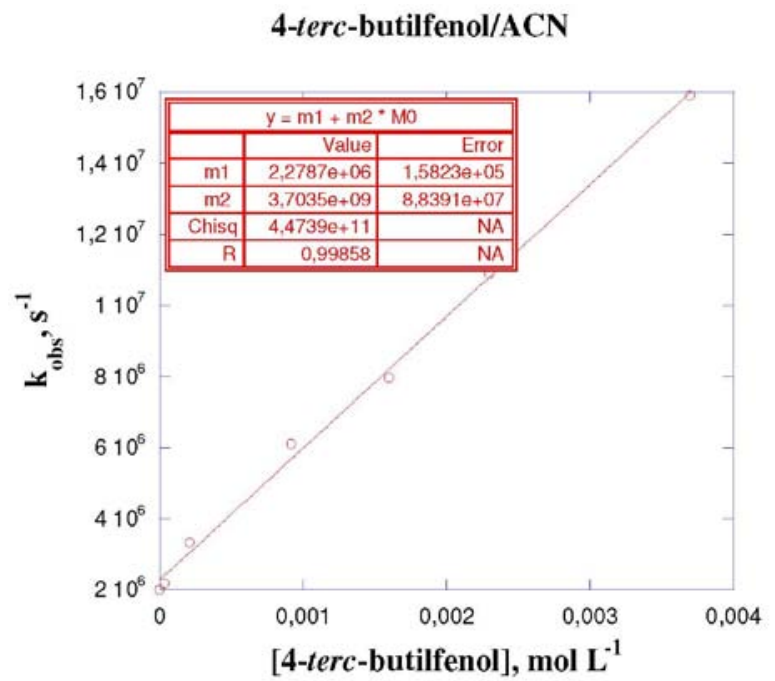

Figura 7S. Gráfico de Stern-Volmer para a supressão do estado excitado triplete de xantona por 4-terc-butilfenol, em acetonitrila

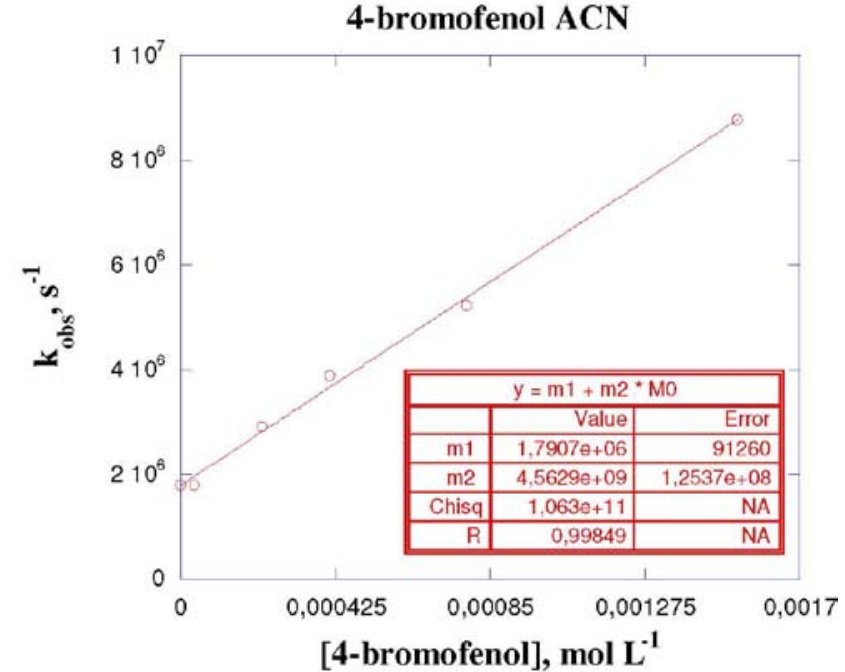

Figura 8S. Gráfico de Stern-Volmer para a supressão do estado excitado triplete de xantona por 4-bromofenol, em acetonitrila

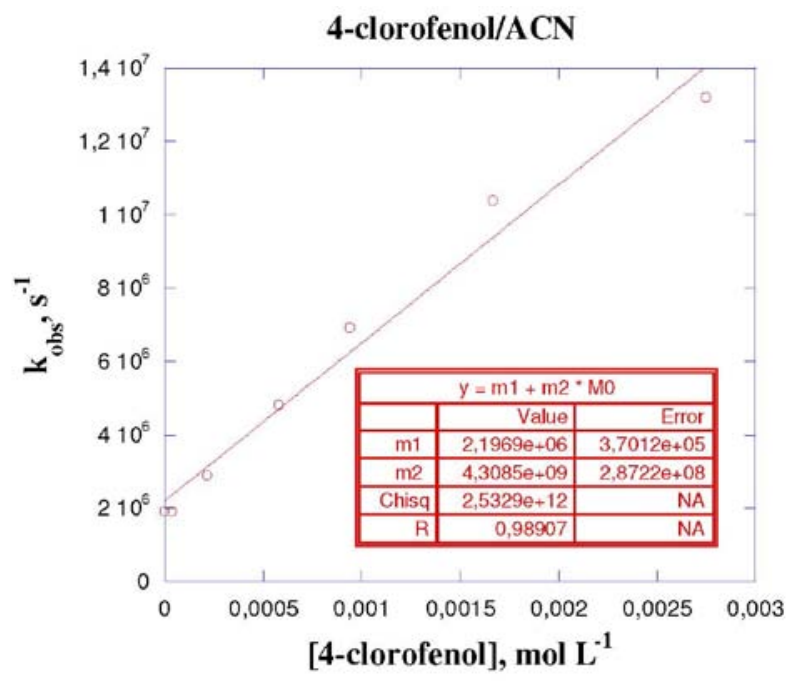

Figura 9S. Gráfico de Stern-Volmer para a supressão do estado excitado triplete de xantona por 4-clorofenol, em acetonitrila

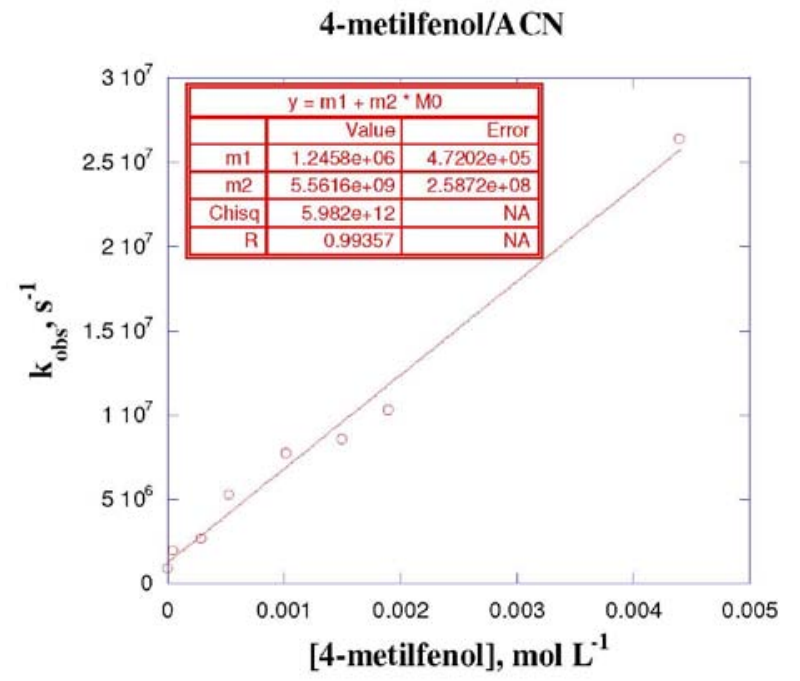

Figura 10S. Gráfico de Stern-Volmer para a supressão do estado excitado triplete de xantona por 4-metilfenol, em acetonitrila 


\section{4-Fenilfenol/ACN}

\begin{tabular}{|c|c|c|c|}
\hline \multirow{2}{*}{$310^{7}$} & \multicolumn{3}{|c|}{$y=m 1+m 2^{*} M 0$} \\
\hline & & Value & Error \\
\hline \multirow[t]{2}{*}{$2,510^{7}$} & $\mathrm{~m} 1$ & $2,1439 \theta+06$ & $3,4845 e+05$ \\
\hline & $\mathrm{m} 2$ & $8 e+09$ & $2,716 e+08$ \\
\hline & Chisq & $1,84 e+12$ & NA \\
\hline $210^{7}$ & $\mathrm{R}$ & 0,9977 & NA \\
\hline
\end{tabular}

"n

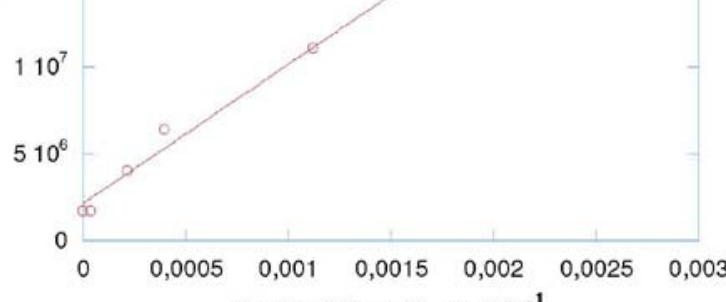

[4-fenilfenol], $\mathrm{mol} \mathrm{L}^{-1}$

Figura 11S. Gráfico de Stern-Volmer para a supressão do estado excitado triplete de xantona por 4-fenilfenol, em acetonitrila

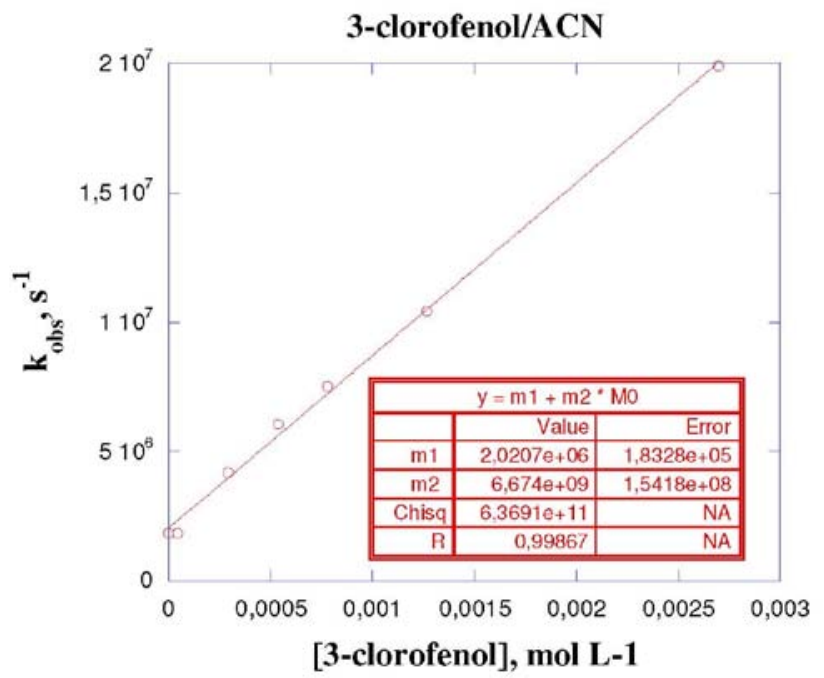

Figura 12S. Gráfico de Stern-Volmer para a supressão do estado excitado triplete de xantona por 3-clorofenol, em acetonitrila

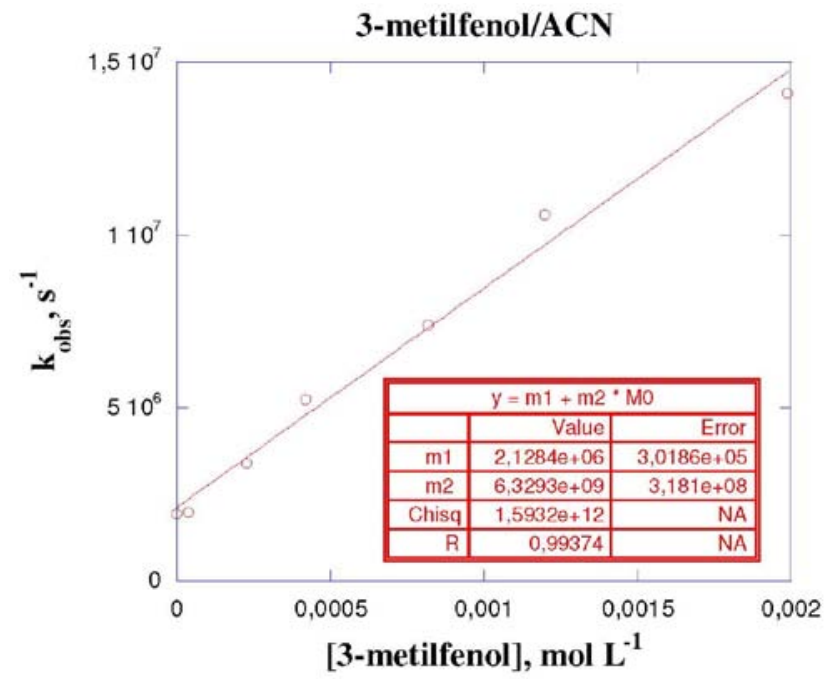

Figura 13S. Gráfico de Stern-Volmer para a supressão do estado excitado triplete de xantona por 3-metilfenol, em acetonitrila

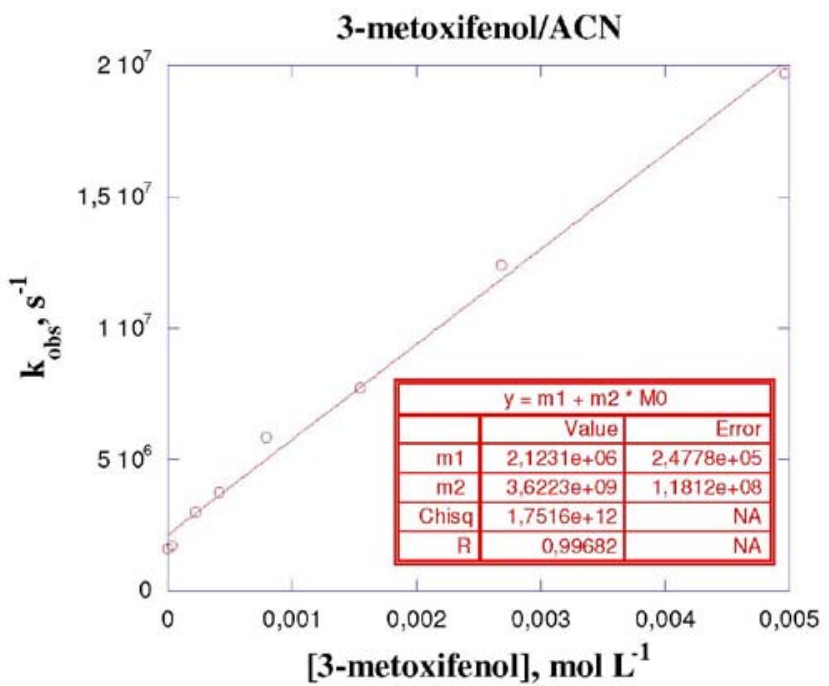

Figura 14S. Gráfico de Stern-Volmer para a supressão do estado excitado triplete de xantona por 3-metoxifenol, em acetonitrila

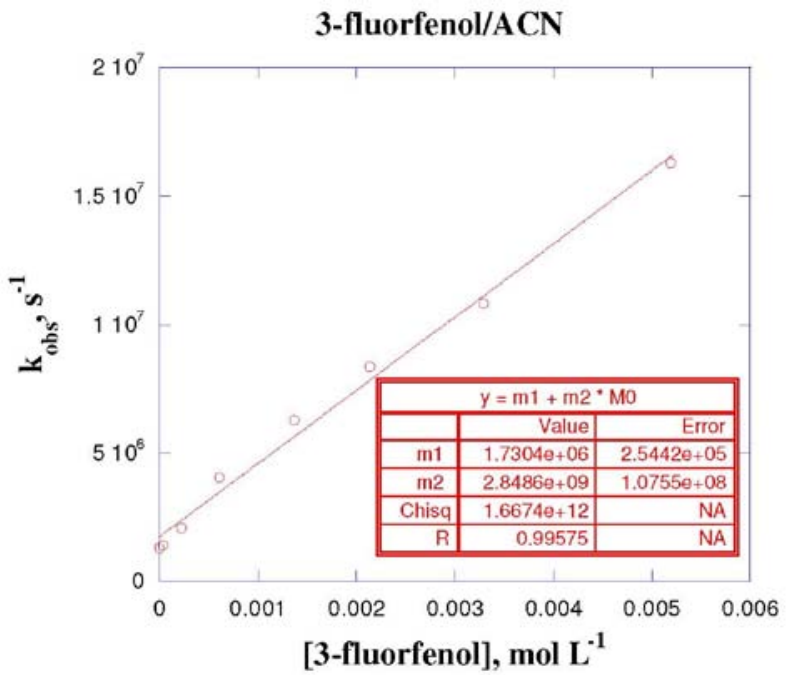

Figura 15S. Gráfico de Stern-Volmer para a supressão do estado excitado triplete de xantona por 3-fluorfenol, em acetonitrila

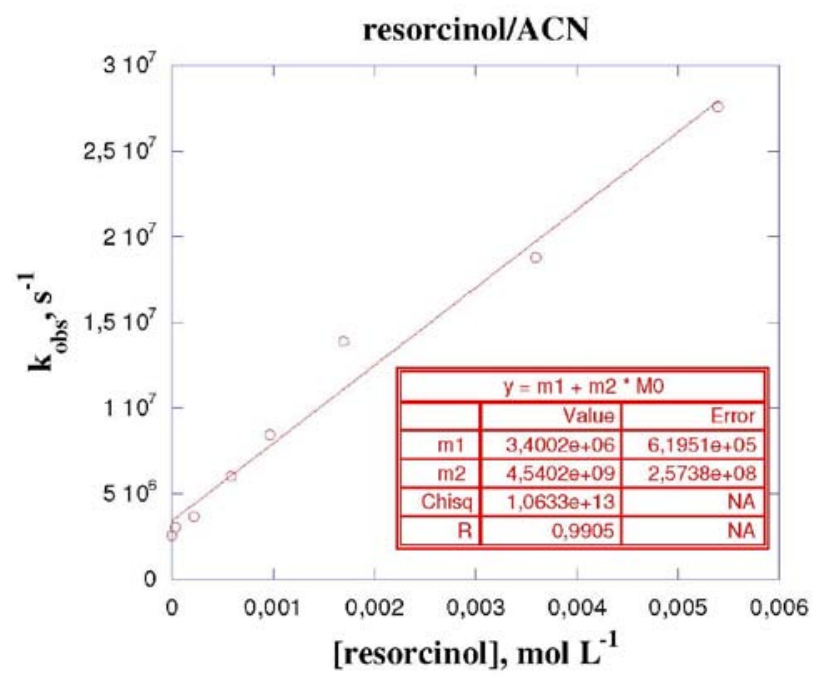

Figura 16S. Gráfico de Stern-Volmer para a supressão do estado excitado triplete de xantona por resorcinol, em acetonitrila 


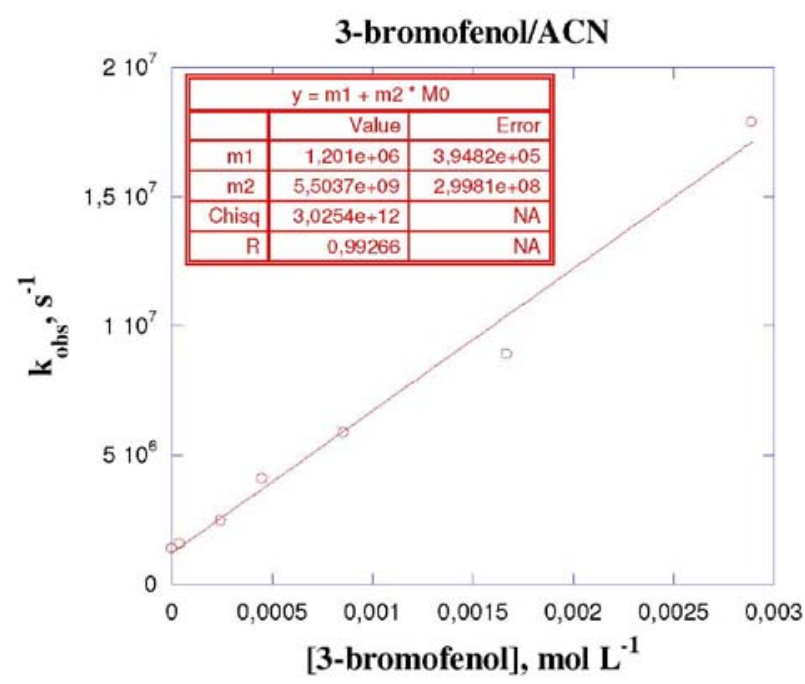

Figura 17S. Gráfico de Stern-Volmer para a supressão do estado excitado triplete de xantona por 3-bromofenol, em acetonitrila

\section{Fenol/BMIM.PF6}

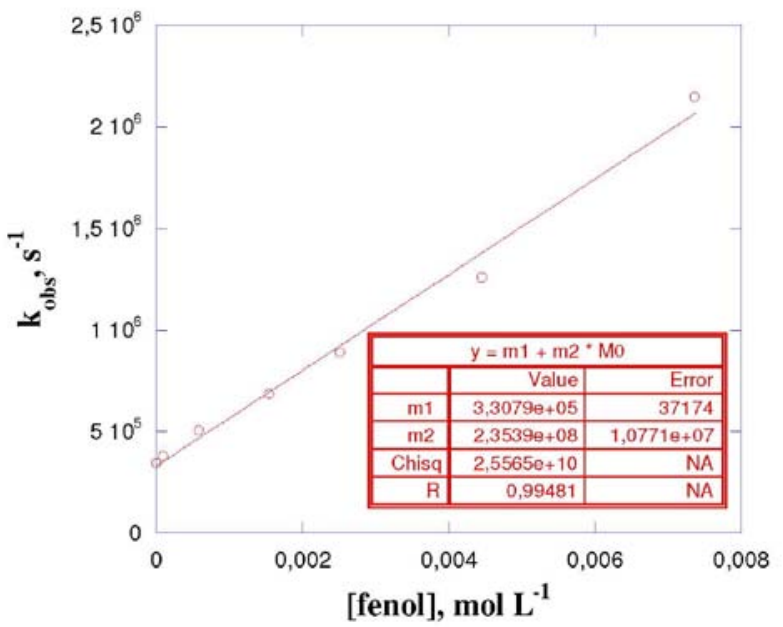

Figura 18S. Gráfico de Stern-Volmer para a supressão do estado excitado triplete de xantona por fenol, no líquido iônico [bmim.PF $\left.F_{6}\right]$

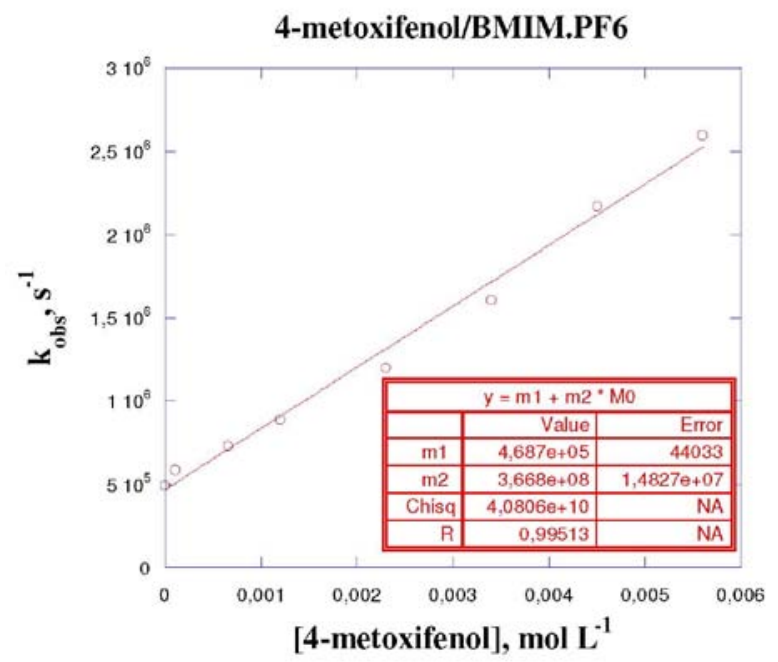

Figura 19S. Gráfico de Stern-Volmer para a supressão do estado excitado triplete de xantona por 4-metoxifenol, no líquido ionico [bmim.PF $\left.F_{6}\right]$

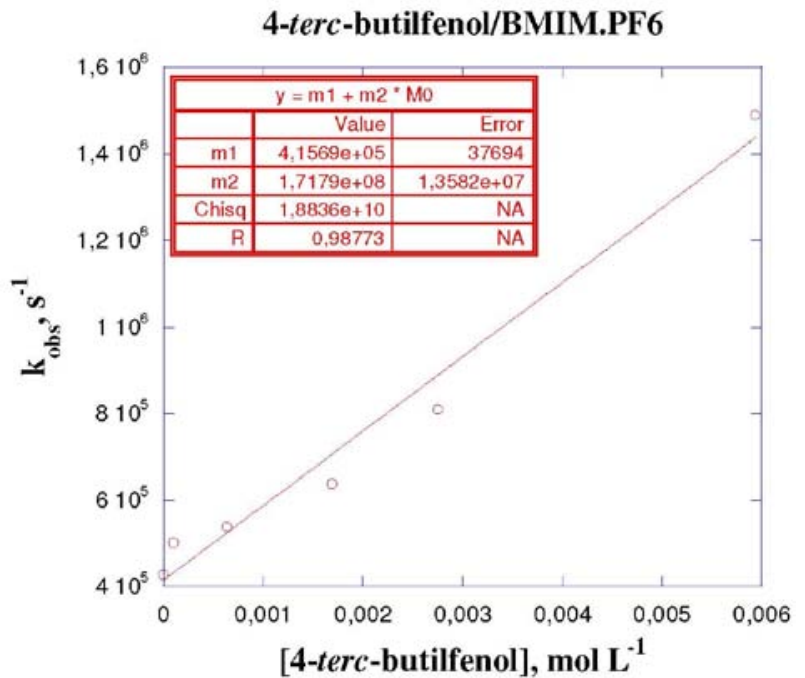

Figura 20S. Gráfico de Stern-Volmer para a supressão do estado excitado triplete de xantona por 4-terc-butilfenol, no líquido ionico [bmim.PF $\left.F_{6}\right]$

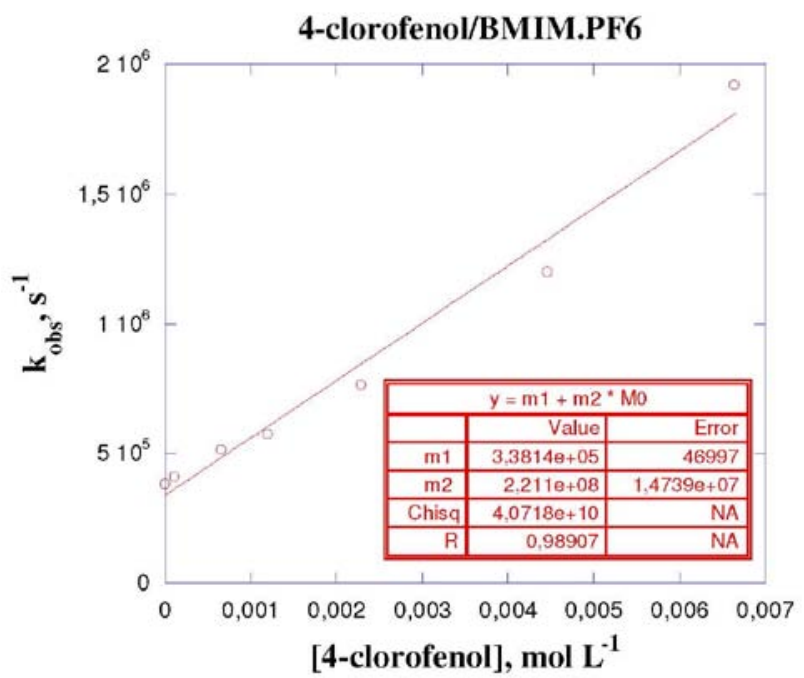

Figura 21S. Gráfico de Stern-Volmer para a supressão do estado excitado triplete de xantona por 4-clorofenol, no líquido iônico [bmim.PF $\left.F_{6}\right]$

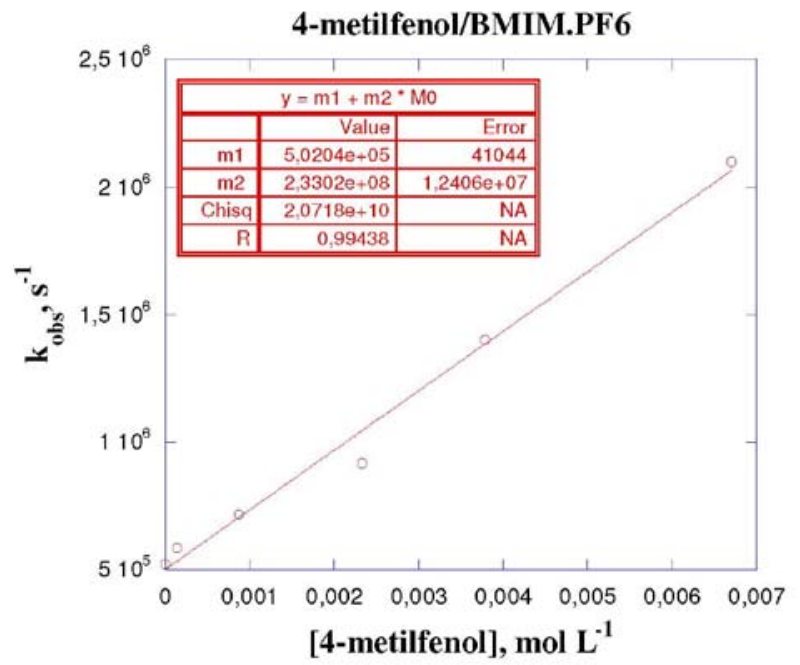

Figura 22S. Gráfico de Stern-Volmer para a supressão do estado excitado triplete de xantona por 4-metilfenol, no líquido iônico [bmim.PF $\left.F_{6}\right]$ 


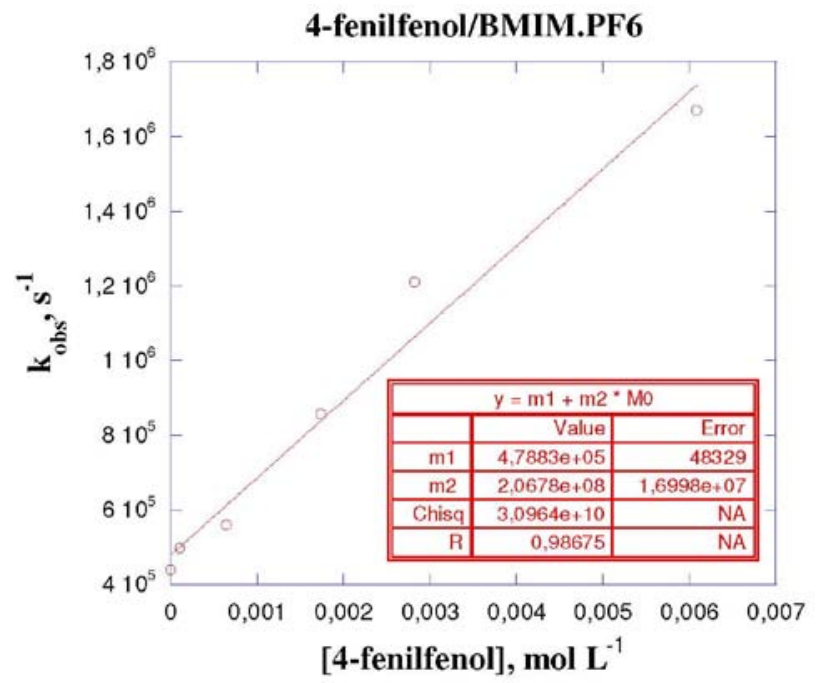

Figura 23S. Gráfico de Stern-Volmer para a supressão do estado excitado triplete de xantona por 4-fenilfenol, no líquido iônico [bmim.PF $\left.F_{6}\right]$ 